PROCEEDINGS OF THE

AMERICAN MATHEMATICAL SOCIETY

Volume 135, Number 10, October 2007, Pages 3083-3090

S 0002-9939(07)08878-8

Article electronically published on June 20, 2007

\title{
THE LINK OF THE GERM OF A SEMI-ALGEBRAIC METRIC SPACE
}

\author{
GUILLAUME VALETTE
}

(Communicated by David Preiss)

\begin{abstract}
In this paper we investigate the metric properties of semi-algebraic germs. More precisely we introduce a counterpart to the notion of link for semi-algebraic metric spaces, which is often used to study the topology. We prove that it totally determines the metric type of the germ. We give a nice consequence for semi-algebraically bi-Lipschitz homeomorphic semi-algebraic germs.
\end{abstract}

\section{INTRODUCTION}

Several authors have manifested interest for the metric type of germs. The study of the link can provide interesting metric invariants ([B], $\mathrm{M}]$, [BB2]). Recently L. Birbrair and J. P. Brasselet have introduced a metric homology [BB1] and studied it in the case of a germ of an isolated singularity.

In [V1] the notion of Lipschitz triangulation is introduced. This tool has been introduced in order to prove a bi-Lipschitz version of Hardt's Theorem. This object gives a counterpart to the notion of triangulations (which usually is devoted to the topology) for the metric type. Here we come back to this notion to describe the metric type of a semi-algebraic germ.

The local conic structure is a well known property of semi-algebraic germs (see BCR1, Theorem 9.3.5). It says that a representative of a semi-algebraic germ is homeomorphic to a cone whose link is the intersection of a "sufficiently small sphere" with our given set. Actually we know that the link is independent of the considered (semi-algebraic) distance function and more generally that it can be defined from the level surface of an arbitrary positive continuous semi-algebraic function satisfying $\rho^{-1}(0)=\{0\}$ (see for instance [CK], Proposition 1). As a matter of fact the link completely characterizes the germ up to a semi-algebraic homeomorphism of the germ and two semi-algebraic homeomorphic subsets can be related by a homeomorphism preserving the distance to the origin.

In this paper we investigate the metric point of view. It means that we will consider the sets up to semi-algebraic bi-Lipschitz homeomorphisms. By considering the two bi-Lipschitz inequivalent cusps $\left\{(x ; y) \in \mathbb{R}^{2} \mid y^{2}=x^{3}\right\}$ and $\left\{(x ; y) \in \mathbb{R}^{2} \mid y^{2}=\right.$ $\left.x^{5}\right\}$, one sees that there may be two subsets not bi-Lipschitz homeomorphic with links semi-algebraically bi-Lipschitz homeomorphic.

Received by the editors September 23, 2005 and, in revised form, July 14, 2006.

2000 Mathematics Subject Classification. Primary 14P10, 32B25, 154E40.

This paper was partially supported by the RAAG Network.

(C)2007 American Mathematical Society Reverts to public domain 28 years from publication 
Therefore we have to take the generic fiber of the distance function that lies in the field of algebraic Puiseux series. The set is morally the same but in some sense it attaches a valuation to points that also determines the metric vanishing of the link on approach to the origin.

We will prove that this link is independent of the distance function (provided it is semi-algebraic Lipschitz and comparable with the euclidian distance), completely determines the metric type (Theorem 0.1) and as a consequence we will see that two semi-algebraic subsets semi-algebraically bi-Lipschitz homeomorphic can be related by a semi-algebraic bi-Lipschitz homeomorphism preserving the distance to the origin (Corollary 0.2).

As the local conic structure of a simplicial complex is rather clear, the notion of Lipschitz triangulation seems to be well adapted to our present problem.

The paper is organized as follows. We first fix the main notations and state the main results. Then in Section 1 we recall the notion of Lipschitz triangulations introduced in V1] and prove a theorem of existence devoted to semi-algebraic germs (Theorem 1.7). In the second section we prove that the link is independent of the choice of the distance function and then we end by proving the main results.

0.1. Notations and main results. Given two germs of semi-algebraic sets $A$ and $B$ we will write $A \simeq B$ if there exists a semi-algebraic bi-Lipschitz homeomorphism between these two sets. Given two positive functions $f$ and $g$ we will write $f \sim g$ if there exist two strictly positive constants $c$ and $C$ such that $c g \leq f \leq C g$.

We will denote by $k\left(0_{+}\right)$the field of real algebraic Puiseux series. We order this field as usual, that is, making the indeterminate $X\left(0_{+}\right)$smaller than any positive real number. It is well known that it constitutes a real closed field (see [BCR12], Example 1.1.2). This field is not archimedean, nevertheless all the Lipschitz constants in this paper will be assumed to be positive real numbers.

As usual given a semi-algebraic subset $A \subseteq \mathbb{R} \times \mathbb{R}^{n}$ we will consider it as a family with the first variable as a parameter. We will denote by $A_{k\left(0_{+}\right)}$the corresponding subset of $k\left(0_{+}\right) \times k\left(0_{+}\right)^{n}$ defined by the same equations regarded in $k\left(0_{+}\right)$. Also we set

$$
A_{0_{+}}=\left\{x \in k\left(0_{+}\right)^{n} \mid\left(X\left(0_{+}\right) ; x\right) \in A_{k\left(0_{+}\right)}\right\}
$$

for the fiber at $0_{+}$of the family $A$ (again see [BCR1-2]) which is actually the fiber at the parameter $X\left(0_{+}\right)$of the family $A$.

Fix $n \in \mathbb{N}$. We will denote by $r$ the euclidian distance to the origin in $\mathbb{R}^{n}$. For $C \in \mathbb{R}$ let us set $\mathcal{C}_{n}(C)=\left\{x=\left(x_{1} ; \ldots ; x_{n}\right) \in \mathbb{R}^{n} / r(x) \leq C x_{1}\right\}$.

Let $\rho$ be a positive semi-algebraic Lipschitz function satisfying $\rho^{-1}(0)=\{0\}$ and

$$
\rho(x) \sim r(x) .
$$

It will be convenient to replace $\mathbb{R}^{n}$ by the graph of $r$ (which is bi-Lipschitz homeomorphic). Thus given a semi-algebraic subset $A$ of $\mathbb{R}^{n}$ we denote

$$
\widetilde{A}=\left\{\left(x_{1} ; q\right) \in \mathbb{R} \times A / x_{1}=r(q)\right\}
$$

the graph of the function $r$ over $A$. Note that $\widetilde{A}$ is a subset of $\mathcal{C}_{n+1}(\sqrt{2})$.

We also define the link of a semi-algebraic subset $A$ for a function $\rho$ satisfying (0.1) as

$$
L_{A}^{\rho}=\left\{x \in A_{k\left(0_{+}\right)} / \rho(x)=X\left(0_{+}\right)\right\}=\widetilde{A}_{0_{+}} .
$$


Whenever $\rho=r$ we will write $L_{A}$ instead of $L_{A}^{r}$. We are going to see that actually the metric type of $L_{A}^{\rho}$ is independent on $\rho$ and completely determines that of $A$. In particular we will prove

Theorem 0.1. Let $A$ and $B$ be germs of semi-algebraic subsets of $\mathbb{R}^{n}$. We have

$$
L_{A} \simeq L_{B} \quad \text { iff } \quad A \simeq B .
$$

This result has the following interesting corollary:

Corollary 0.2. Two semi-algebraic subsets which are semi-algebraically biLipschitz homeomorphic can be related by a semi-algebraic bi-Lipschitz homeomorphism preserving the distance to the origin.

\section{LiPSCHITZ TRIANGULATIONS}

In this section we recall the notion of "Lipschitz triangulation". As we shall need to triangulate only germs of semi-algebraic sets the definition will be a bit simpler than that of [V1]. Namely the functions $\varphi_{i, \sigma}$ in (1.3) do not depend on $q^{\prime}$.

The object is very natural. It is impossible to construct a triangulation that is a bi-Lipschitz homeomorphism. This is because the notion of contact is crucial to describe the metric type. This contact is expressed by contractions operating on coordinates over each simplex. These contractions are just sums of products of powers of distances to faces. That is a finite sum of functions of the following form:

$$
d\left(q ; \sigma_{1}\right)^{\alpha_{1}} \cdot \ldots \cdot d\left(q ; \sigma_{k}\right)^{\alpha_{k}}
$$

where $\sigma_{1}, \ldots, \sigma_{k}$ are simplices of $K$ and $\alpha_{1}, \ldots, \alpha_{k}$ are real numbers.

We call a finite sum of such functions a "standard simplicial function".

The second thing we shall need is what we call the tame systems of coordinates, in order to express the directions along which contractions operate:

Definition 1.1. A tame system of coordinates on $\mathbb{R}^{n}$ is a family of functions $\left(\psi_{1} ; \ldots ; \psi_{n}\right)$ of the following form:

$$
\psi_{i}(q)=\frac{q_{i}-\theta_{i}\left(\pi_{i-1}(q)\right)}{\theta_{i}\left(\pi_{i-1}(q)\right)-\theta_{i}^{\prime}\left(\pi_{i-1}(q)\right)}
$$

(and 0 whenever $\theta_{i} \circ \pi_{i-1}(q)=\theta_{i}^{\prime} \circ \pi_{i-1}(q)$ ) where $\theta_{i}$ and $\theta_{i}^{\prime}$ are piecewise linear functions on $\mathbb{R}^{i-1}$.

Definition 1.2. A Lipschitz triangulation of a germ $\left(\mathbb{R}^{n} ; 0\right)$ is the data of a germ of a finite simplicial complex $K$ together with a germ of homeomorphism $h:|L| \rightarrow \mathbb{R}^{n}$, where $L$ is a union of open simplices of $K$, such that for every $\sigma \in L$ there exist $\varphi_{\sigma, 1}, \ldots, \varphi_{\sigma, k}$, standard simplicial functions over $\sigma \times \sigma$ satisfying for any $q$ and $q^{\prime}$ in $\sigma$ :

$$
\left|h(q)-h\left(q^{\prime}\right)\right| \sim \sum_{i=1}^{n} \varphi_{\sigma, i}(q) \cdot\left|q_{i, \sigma}-q_{i, \sigma}^{\prime}\right|
$$

where $\left(q_{1, \sigma}, \ldots, q_{n, \sigma}\right)$ is a piecewise linear system of coordinates of $\mathbb{R}^{n}$. Let $A_{1}, \ldots$, $A_{k}$ be germs of subsets of $\mathbb{R}^{n}$. A Lipschitz triangulation of $A_{1}, \ldots, A_{k}$ is a Lipschitz triangulation of $\mathbb{R}^{n}$ such that each $h^{-1}\left(A_{i}\right)$ is a union of open simplices. 
1.1. Regular directions. The main difficulty in the construction of such an object is to find a regular direction. This means that we need to find a line that is not contained in any limit of tangent space. This is impossible in general but in [V1] it is proved that we can obtain it up to a bi-Lipschitz homeomorphism. We improve it in the particular setting of the present paper by proving that, for a subset of $\mathcal{C}_{n}(C)$, we may find a homeomorphism that preserves the first coordinate as well. In the case of $L_{A}$ this will enable us to construct a homeomorphism preserving the distance to the origin.

Definition 1.3. Let $A$ be a semi-algebraic set of $\mathbb{R}^{n+1}$. An element $\lambda$ of $S^{n}$ is said to be regular for $A$ if there is a strictly positive real number $\alpha$ such that

$$
d\left(\lambda ; T_{x} A\right) \geq \alpha
$$

for all $x$ in the regular locus of $A$.

In the following proposition $R$ denotes a real closed field. This proposition is proven in V1.

Proposition 1.4 ([V1]). Let $A$ be a semi-algebraic subset of $R^{n+1}$ of empty interior. Then there exists a semi-algebraic bi-Lipschitz homeomorphism $h: R^{n+1} \rightarrow$ $R^{n+1}$ such that $e_{n+1}$ is regular for $h(A)$.

In the present setting we can extend the previous proposition into the following lemma (which is actually deduced from the previous proposition).

Lemma 1.5. Let $(A ; 0)$ be the germ of a semi-algebraic subset $\mathcal{C}_{n+1}(C)$, for some $C$, of empty interior. Then there exists a germ of semi-algebraic bi-Lipschitz homeomorphism $h$ such that $e_{n+1}$ is regular for $h(A)$,

$$
\pi_{1}(h(x))=x_{1} .
$$

Proof. Apply Proposition 1.4 to $A_{0_{+}}$where $R=k\left(0_{+}\right)$. This provides a germ of homeomorphism $h: \mathbb{R}^{n+1} \rightarrow \mathbb{R}^{n+1}$ satisfying (1.4) such that $e_{n+1}$ is regular for $h\left(A_{x_{1}}\right)$, for $x_{1}$ small enough. We now have to check that $e_{n+1}$ is regular for the germ of $h(A)$. Suppose not. Then there exists a semi-algebraic arc $\gamma:\left[0 ; \epsilon\left[\rightarrow A_{\text {reg }}\right.\right.$ with $\gamma(0)=0$ and $e_{n+1} \in \tau=\lim T_{\gamma(t)} A_{\text {reg }}$. But as $e_{n+1}$ is regular for the fibers of $A_{x_{1}}$ we have $e_{n+1} \notin \lim T_{\gamma(t)} A_{\gamma_{1}(t)}$. This implies that $\tau \cap\left\langle e_{1}\right\rangle^{\perp} \neq \lim \left(T_{\gamma(t)} A_{r e g} \cap\left\langle e_{1}\right\rangle^{\perp}\right)$ and so $\tau$ is not transverse to $\left\langle e_{1}\right\rangle^{\perp}$ which means that $\tau \subseteq\left\langle e_{1}\right\rangle^{\perp}$. This implies that the limit of the secant to the origin $\lim \frac{\gamma}{|\gamma|}=\lim \frac{\gamma^{\prime}}{\left|\gamma^{\prime}\right|}$ is orthogonal to $e_{1}$. Therefore

$$
\lim \frac{\gamma_{1}(t)}{|\gamma(t)|}=0
$$

in contradiction with $\gamma(t) \in \mathcal{C}_{n}(C)$.

1.2. Lipschitz triangulations: A local version. In this section we give a proof of existence of Lipschitz triangulations for semi-algebraic germs. As our study is local we are able to give a more precise result than in V1. Actually we will triangulate $\widetilde{A}$ preserving the first coordinate. This implies that the link is triangulated (it is the section by the plane $\left\{x_{1}=X\left(0_{+}\right)\right\}$) which is important in the point of view of the local conic structure.

First we recall a proposition, proved in [V1]: 
Proposition 1.6. Let $\xi: \mathbb{R}^{n} \rightarrow \mathbb{R}$ be a positive semi-algebraic function. Then there exists a partition of $\mathbb{R}^{n}$ such that over each element of this partition the function $\xi$ is $\sim$ to a product of powers of distances to semi-algebraic subsets of $\mathbb{R}^{n}$.

Semi-algebraic subsets turn out to present the significant advantage of being triangulable in our meaning. Here we present a result for germs with a specific property.

Theorem 1.7 (compare with [V1]). Let $(A ; 0)$ be semi-algebraic germ of $\mathbb{R}^{n+1}$. Then there exists a semi-algebraic Lipschitz triangulation of $\widetilde{A}$ satisfying

$$
\pi_{1}(h(x))=x_{1} .
$$

Proof. The proof is modeled on the proof of [V1], Theorem 5.1.3 (the reader who needs more details may refer to this article). We have just to check that (1.5) can be obtained in the present setting. More precisely we shall prove inductively on $n$ the following statements:

$\left(H_{n}\right)$ Given a finite number of semi-algebraic sets $A_{1}, \ldots, A_{m}$ of $\mathcal{C}_{n}(C)$ there exists a Lipschitz triangulation of the sets $A_{i}$ satisfying (1.4). Moreover given a finite number of semi-algebraic functions $\eta_{l}$ this can be obtained in such a way that each of these functions is $\sim$ to a sum of products of powers of distances to faces on each simplex.

As $\widetilde{A}$ is a subset of $\mathcal{C}_{n+1}(\sqrt{2})$, the conclusion follows.

The assertion $\left(H_{0}\right)$ is obvious. Assume that it is true at $n$. We may assume that the subsets $A_{1}, \ldots, A_{k}$ are of empty interior since it is enough to construct a triangulation of their boundaries. Apply Proposition 1.5 to the set $\bigcup_{i=1}^{m} A_{i}$. We thus get a subset $L_{A}^{\prime} \in \mathcal{C}_{n+1}(C)$, for some $C$, which can be included into a finite number of graphs of some semi-algebraic Lipschitz functions $\xi_{1} \leq \cdots \leq \xi_{m}$. Then we construct our Lipschitz triangulation as in V1]. We denote by $\pi: \mathbb{R}^{n+1} \rightarrow \mathbb{R}^{n}$ the canonical projection.

Apply Proposition 1.6 to each function $\eta_{l}$ to have a partition $\left(V_{i}\right)_{i \in I}$ of $\mathcal{C}_{n}(C)$ such that, over each $V_{i}$, each function $\eta_{l}$ is equivalent to a product of powers of distances to semi-algebraic subsets $\left(W_{j}\right)_{j \in J}$ of $\mathcal{C}_{n}(C)$. By Proposition 1.5 we may assume that all the boundaries of the elements of the families $\left(V_{i}\right)_{i \in I},\left(A_{i}\right)_{1 \leq i \leq k}$ and $\left(W_{i}\right)_{i \in J}$ are included in the union of a finite number of graphs of some Lipschitzian functions $\theta_{1} \leq \cdots \leq \theta_{\mu}$.

Consider a cylindrical semi-algebraic cell decomposition of $\mathbb{R}^{n+1}$ adapted to the graphs of the functions $\xi_{i}$ and the sets $W_{i}$. This provides a partition $X_{1}, \ldots, X_{s}$ of $\mathbb{R}^{n}$.

Apply the induction hypothesis to get a Lipschitz triangulation $(h ; K)$ of the sets $X_{i}, 1 \leq i \leq s$.

Also, again by the induction hypothesis, we may do it in such a way that over each simplex, each function $\left|\xi_{j}-\theta_{i}\right| \circ h$ and all the functions $q \rightarrow d\left(h(x) ; \pi\left(\partial W_{j} \cap \Gamma_{\theta_{i}}\right)\right)$ are $\sim$ to standard simplicial functions, where $\Gamma_{\theta_{i}}$ is the graph of $\theta_{i}$.

The simplicial complex is constructed as for topological triangulations (see [C], $[\underline{\mathrm{S}})$. Let $\zeta_{1} \leq \cdots \leq \zeta_{m}$ be piecewise linear functions over $|K|$ such that $\zeta_{i}=\zeta_{i+1}$ whenever $\xi_{i} \circ h=\bar{\xi}_{i+1} \circ h$. Let

$$
N=\left\{\left(x ; q_{n+1}\right) \in \mathbb{R}^{n} \times \mathbb{R} / \zeta_{1}(x) \leq q_{n+1} \leq \zeta_{m}(x)\right\} .
$$

We obtain a polyhedral decomposition of $N$ by taking the inverse image by $\pi_{\mid N}$ of the simplices of $K$ of dimension $n-1$ on the one hand, and by taking all the 
images of $|K|$ by the mappings $x \rightarrow\left(x ; \zeta_{i}(x)\right)$ on the other hand. After a barycentric subdivision of this polyhedral we obtain a simplicial complex $\widetilde{K}$.

The union of the graphs gives a simplicial complex. Let $\widetilde{L}$ be the union of open simplices $\sigma$ lying over $|K|$ and included in $N$.

Now define over $\widetilde{L}$ the desired homeomorphism $\widetilde{h}$ in the following way:

$$
\widetilde{h}\left(x ; t \zeta_{i}(x)+(1-t) \zeta_{i+1}(x)\right)=\left(h(x) ; t \xi_{i}(h(x))+(1-t) \xi_{i+1}(h(x))\right)
$$

for $1 \leq i \leq m, x \in \mathbb{R}^{n}$ and $t \in[0 ; 1]$.

This defines a homeomorphism $\widetilde{h}:|\widetilde{L}| \rightarrow \mathbb{R}^{n+1}$.

It remains to check that over each simplex $\sigma$ the mapping $\widetilde{h}$ fulfills an inequality of type (1.3). This can be done exactly as in [V1]. As the subsets $W_{i}$ have been triangulated, it can also be seen (see again [V1]) that the functions $x \rightarrow d\left(h(x) ; W_{i}\right)$ (and so the functions $\eta_{l}$ ) are $\sim$ to standard simplicial functions.

Remark 1. In the situation of the above proposition it is possible to assume the following extra property: the angle between the tangent line to the rays $h(0 q)$ and the secants $0 h(q)$ tends to zero when $q$ tends to zero. This will be useful for Corollary 2.2 .

\section{ON THE LINK OF A SEMI-ALGEBRAIC GERM}

In this section we start to investigate the metric type of a semi-algebraic germ. We prove that the link is independent on the function $\rho$ (provided it is Lipschitz and it satisfies (0.1) $)$. Then we deduce Theorem 0.1 and Corollary 0.2 . We first prove a preliminary lemma.

For simplicity we set $S_{0_{+}}^{\rho}=\rho^{-1}\left(X\left(0_{+}\right)\right)$.

Lemma 2.1. Let $\rho$ be a Lipschitz semi-algebraic function satisfying (0.1). Suppose that for a couple $(p ; q) \in S_{0_{+}}^{\rho}$ we have

$$
|p-q| \leq \varepsilon|p|
$$

for any strictly positive real constant $\varepsilon$. Then the angle between the secant $p q$ and the ray $0 q$ is bounded below by a strictly positive element of $\mathbb{R}$.

Proof. We have to be careful since the function $\rho$ is not necessarily smooth. We thus will consider its derivative as existing generically. We first note that, thanks to the assumptions we made on this function, the limit of $\frac{\partial \rho}{|\partial \rho|}$ along an analytic path tending to zero cannot be orthogonal to the limit of the secant (of the considered path). Otherwise parameterizing $\gamma$ by the distance to the origin we would have

$$
\frac{d \rho(\gamma(r))}{d r}=\partial_{\gamma(t)} \rho \cdot \gamma^{\prime}(r)
$$

which would tend to zero. This would contradict (0.1).

This implies that the inner product $\partial_{q} \rho . q$ is around the origin bounded away from zero and so is positive since $\rho$ is positive and $\rho(0)=0$.

Now we assume that the conclusion of the proposition fails. So we get two semialgebraic arcs $p(t)$ and $q(t)$ ending at the origin such that the secant between them has the same limit (in the projective space) as the secant to the origin. Slightly moving $q$ to $q_{1}$ we may assume that $\rho$ is differentiable (almost everywhere) on the secant $p q_{1}$. Note that by the above observation the inner product $\partial_{x} \rho . p q_{1}$ is bounded below from zero (by a constant independent on the choice of $q_{1}$ sufficiently closed 
to $q$ ) for $x$ (almost everywhere) on the segment $p q_{1}$ and so $\rho$ is strictly monotonic on the secant. This contradicts that the points $p(t)$ and $q(t)$ belong to the same level surface of $\rho$.

Corollary 2.2. Up to a semi-algebraic bi-Lipschitz homeomorphism, the link $L_{A}$ is independent of the semi-algebraic Lipschitz function $\rho$ used to define it (provided (0.1) is fulfilled).

Proof. It is enough to construct a bi-Lipschitz map $g: \widetilde{S}_{0_{+}}^{\rho} \rightarrow \widetilde{S}^{(n-1)}$ preserving $\widetilde{A}_{k\left(0_{+}\right)} \cdot$

Let $h:|K| \rightarrow \mathbb{R}^{n+1}$ be the mapping given by Theorem 1.7 applied to the germ $A$. First we define a linear retraction onto the fiber at $X\left(0_{+}\right) \mu: k\left(0_{+}\right)^{*} \times k\left(0_{+}\right)^{n} \rightarrow$ $k\left(0_{+}\right)^{n+1}$ by setting $\mu\left(x_{1} ; x^{\prime}\right)=\left(X\left(0_{+}\right) ; \frac{X\left(0_{+}\right)}{x_{1}} x^{\prime}\right)$. Note that $\mu$ preserves the germs of simplicial complexes and, in the situation of Theorem 1.7, the tame coordinates $q_{i, \sigma}$ for $i \geq 2$.

Now for every $c, C \in \mathbb{R}$ there exists a semi-algebraic homeomorphism

$$
\Phi: \mathcal{C}_{n}(\sqrt{2})_{k\left(0_{+}\right)} \backslash\{0\} \rightarrow\left[c X\left(0_{+}\right) ; C X\left(0_{+}\right)\right] \times k\left(0_{+}\right)^{n}
$$

preserving the points of $\widetilde{A}_{k\left(0_{+}\right)}$. It suffices to set $\Phi(x)=\left(x_{1} ; h \circ \mu \circ h^{-1}(x)\right)$. As $\mu$ preserves the tame coordinates, by (1.3) and (1.4), $\Phi$ is a Lipschitz map and that $\Phi_{x_{1}}$ (the restriction of the mapping for a fixed $x_{1}$ ) is bi-Lipschitz with a uniform constant.

Now write $\Phi\left(x_{1} ; y\right)=\left(\theta ; x_{1}\right)$ in $\left[c X\left(0_{+}\right) ; C X\left(0_{+}\right)\right] \times k\left(0_{+}\right)^{n}$. Let $g=\theta_{\mid \widetilde{S}^{\rho}\left(X\left(0_{+}\right)\right)}$. Clearly $g$ is a Lipschitz homeomorphism and as $\mu$ preserves the simplices $g$ preserves $\widetilde{A}$. Let $q=\left(x_{1} ; y\right)$ and $q^{\prime}=\left(x_{1}^{\prime} ; y^{\prime}\right)$ satisfy $\widetilde{\rho}(h(q))=\widetilde{\rho}\left(h\left(q^{\prime}\right)\right)=X\left(0_{+}\right)$. Let $x=\frac{x_{1}}{x_{1}^{\prime}}$. We claim that for such points $q$ and $q^{\prime}$

$$
\left|h(q)-h\left(q^{\prime}\right)\right| \sim\left|h(q)-h\left(x q^{\prime}\right)\right| .
$$

By Remark 1 we know that the angle between $h(q) h(x q)$ and $0 h(q)$ is less than any positive element of $\mathbb{R}$. As $h(q)$ and $h\left(q^{\prime}\right)$ are in $\widetilde{S}_{0_{+}}^{\rho}$, by Lemma 2.1 we deduce that the secant lines $h(q) h\left(q^{\prime}\right)$ and $h(q) h(x q)$ have an angle bounded below from zero by an element of $\mathbb{R}$. The claim follows.

Since the image of $x q$ by $\mu$ is that of $q^{\prime}$ and $\Phi_{x_{1}}$ is bi-Lipschitz we see that the above claim implies that $g$ is bi-Lipschitz.

Remark 2. The mapping $\Phi$ constructed in the previous proof is actually a semialgebraic bi-Lipschitz homeomorphism.

Proof of Theorem 0.1 and Corollary 0.2. "if." Let $h: A \rightarrow B$ be a bi-Lipschitz homeomorphism. Let $\rho=r \circ h$. Then $L_{A}^{\rho} \simeq L_{B}$ and by the above proposition $L_{B} \simeq L_{A}$.

"only if." Let $h: k\left(0_{+}\right)^{n} \rightarrow k\left(0_{+}\right)^{n}$ such that $h\left(L_{A}\right)=L_{B}$ is a bi-Lipschitz homeomorphism. Then by the Tarski-Seidenberg principle this induces a germ homeomorphism $g: \mathbb{R}^{n} \rightarrow \mathbb{R}^{n}$, preserving the distance to the origin, sending $A$ onto $B$ and which is bi-Lipschitz on each sphere (with a uniform Lipschitz constant). We have to check that it is also Lipschitz with respect to $x_{1}$ (by symmetry it is enough to prove it is Lipshitz). Suppose not. Then we can find two $\operatorname{arcs}$ in $A$, say $p(t)$ and $q(t)$, tending to zero along which it is not Lipschitz.

In particular we have

$$
|| p|-| q|| \ll|h(p)-h(q)| .
$$


Recall that $h$ preserves the distance to the origin. We may parameterize $p(t)$ (and so $h(p(t)))$ by its distance to the origin. As the tangent to the two curves $p(t)$ and $h(p(t))$ has the same limit as their respective secant lines to the origin we have

$$
\left|h(p(r))-h\left(p\left(r^{\prime}\right)\right)\right| \sim\left|r-r^{\prime}\right|
$$

and

$$
\left|p(r)-p\left(r^{\prime}\right)\right| \sim\left|r-r^{\prime}\right| .
$$

Therefore by (2.1) and (2.2) we have $|h(p(r))-h(q(r))| \sim\left|h\left(p\left(r^{\prime}\right)\right)-h(q(r))\right|$, where $r^{\prime}$ denotes the distance of $q$ to the origin, and now we get a contradiction from (2.3) and from the Lipschitzness of $h$ in restriction to the the spheres.

By Theorem 0.1 when two sets are semi-algebraically bi-Lipschitz homeomorphic they have semi-algebraically bi-Lipschitz homeomorphic links and by the same arguments as in the proof of the "only if" condition the homeomorphism induces a bi-Lipschitz homeomorphism preserving the distance to the origin. This proves Corollary 0.2 .

\section{REFERENCES}

[B] L. Birbrair, Local bi-Lipschitz classification of 2-dimensional semialgebraic sets. Houston J. Math. 25 (1999), no. 3, 453-472. MR.1730886(2000j:14091)

[BB1] L. Birbrair, J. P. Brasselet, Metric homology. Comm. Pure Appl. Math. 53 (2000), no. 11, 1434-1447. MR 1773415 (2001h:14075)

[BB2] L. Birbrair, J. P. Brasselet, Metric homology for isolated conical singularities. Bull. Sci. Math. 126 (2002), no. 2, 87-95. MR1906238(2003d:14070)

[BCR1] J. Bochnak, M. Coste, M-F. Roy, Géométrie Algébrique Réelle, Ergebnisse der Math. 12, Springer-Verlag (1987). MR949442 (90b:14030)

[BCR2] J. Bochnak, M. Coste, M-F. Roy, Real Algebraic Geometry, Ergebnisse der Math. 36, Springer-Verlag (1998). MR.1659509 (2000a:14067)

[C] M. Coste, An introduction to O-minimal Geometry, Dip. Mat. Univ. Pisa, Dottorato di Ricerca in Matematica, Instituti Editoriali e Poligrafici Internazionali, Pisa (2000).

[CK] M. Coste, K. Kurdyka, On the link of a stratum in a real algebraic set. Topology 31 (1992), no. 2, 323-336. MR1167174 (93d:14088)

[vDS] L. van den Dries, P. Speissegger. O-minimal preparation theorems. Model theory and applications, 87-116, Quad. Mat., 11, Aracne, Rome, 2002. MR2159715 (2006m:03063)

[M] T. Mostowski, Lipschitz equisingularity. Dissertationes Math. (Rozprawy Mat.) 243 (1985), 46 pp. MR808226 (87e:32008)

[S] M. Shiota, Geometry of subanalytic and semialgebraic sets. Progress in Mathematics, 150. Birkhäuser Boston, Inc., Boston, MA, 1997. MR1463945 (99b:14061)

[V1] G. Valette, Lipschitz triangulations. Illinois J. of Math., Vol. 49, No. 3, Fall 2005, 953-979. MR2210270

[V2] G. Valette, Hardt's theorem: A bi-Lipschitz version. C. R. Acad. Sci. Paris, Ser. I 340 (2005), issue 12, 895-900. MR.2152275 (2006a:14097)

Instytut Matematyki, Uniwersytet Jagielloński, Ul. Reymonta 4, 30-059 Kraków, POLAND

E-mail address: Guillaume.Valette@im.uj.edu.pl 